\title{
Single top-quark production with SHERPA
}

\author{
Enrico Bothmann $^{1, \mathrm{a}}$, Frank Krauss ${ }^{2}$, Marek Schönherr ${ }^{3}$ \\ ${ }^{1}$ Higgs Centre for Theoretical Physics, University of Edinburgh, Edinburgh EH9 3FD, UK \\ 2 Institute for Particle Physics Phenomenology, Durham University, Durham DH1 3LE, UK \\ ${ }^{3}$ Theoretical Physics Department, CERN, 1211 Geneva 23, Switzerland
}

Received: 15 November 2017 / Accepted: 3 March 2018 / Published online: 15 March 2018

(C) The Author(s) 2018

\begin{abstract}
We present results at next-to-leading order accuracy in QCD for single top-quark production in the $t, s$ and $t W$ channels at the LHC at a centre-of-mass energy of $8 \mathrm{TeV}$, obtained with the SHERPA event generator. We find them in very good agreement with measured values and quantify their theory uncertainties. Uncertainties stemming from the choice between the four- and the five-flavour scheme are found to be typically of the order of 5-10\% over large ranges of phase space. We discuss the impact of parton distribution functions, and in particular of the bottom PDF. We also show how different cuts on QCD radiation patterns improve the signal-to-background ratio in realistic fiducial volumes.
\end{abstract}

\section{Introduction}

The production of single-top quarks is an important source of backgrounds in searches for new physics [1-3], but it is also a signal in its own right [4-10] since it allows the direct determination of the Cabibbo-Kobayashi-Maskawa (CKM) matrix element $\left|V_{t b}\right|[6,11,12]$. By far and large it has become customary to distinguish three modes for singletop production, differentiated by the role played by the $W$ boson, namely $s$-channel production $\left(q \bar{q}^{\prime} \rightarrow t \bar{b}, \bar{t} b\right.$ at Born level), $t$-channel production $\left(q \bar{b} \rightarrow q^{\prime} \bar{t}\right.$ and $\bar{q} b \rightarrow \bar{q}^{\prime} t$ at Born level), and $t W$-associated production, $\left(g b \rightarrow t W^{-}\right.$and $g \bar{b} \rightarrow \bar{t} W^{+}$at Born-level). Fixed-order predictions at nextto-leading accuracy in QCD (NLO) have been presented for the $s$-channel in $[13,14]$, for the $t$-channel in $[14,15]$, and for $t W$-associated production in [16]. Monte-Carlo simulations that are accurate to NLO have been constructed for all three channels with the Mc@NLO method $[17,18]$ and with the POWHEG method $[19,20]$. Four-flavour scheme variants of these results have been presented and compared with the five-flavour scheme ones in $[21,22]$. Results for the production and subsequent decay of single top-quarks at NLO pre-

\footnotetext{
a e-mail: enrico.bothmann@ed.ac.uk
}

cision for the $s$ and the $t$ channel have been presented in [23], as part of the MCFM package. Furthermore, the cross section for the dominant $t$-channel mode has been calculated up to NNLO in QCD [24-26] and are found to be fully covered by scale variations at NLO accuracy, at the level of 1-2\%. Electroweak corrections to single-top production have been discussed in [27]: In the $s$-channel, and depending on the centerof-mass energy of the partonic system, they range from about $+2 \%$ at $\hat{s}^{1 / 2}=200 \mathrm{GeV}$ to about $-30 \%$ at $\hat{s}^{1 / 2}=7 \mathrm{TeV}$, while in the $t$-channel they are, surprisingly, consistently positive, and range between about $+6 \%$ and $+10 \%$ for the same partonic center-of-mass energies.

In this publication, we present results obtained with the SHERPA event generation framework [28] for single-top production in all three channels. After a short description of the generation setups below, Sect. 2, we will contrast our results with experimentally measured data in Sect. 3. In this section we also further investigate theory uncertainties on typical distributions for the two production channels, with some emphasis on the use of the four- or five-flavour scheme for $t$-channel production. We also comment on the impact of different bottom parton distribution functions (PDFs) on selected observables. Finally, in Sect. 4, we will investigate signal-to-background ratios, especially for $t$-channel production, and how they can be improved through cuts on light jets, before summarising our findings in Sect. 5 .

\section{Setup}

We calculate all three single-top production channels, the $t$-, the $s$ - and the $t W$-channel at a centre-of-mass energy of $\sqrt{s}=8 \mathrm{TeV}$, using the Mc@ NLO technique $[17,29]$ in the variant implemented in SHERPA, S-Mc@NLO [30-32]. Up to NLO QCD the $t$ - and $s$-channel process are unequivocally defined by the presence of a $t$ - and $s$-channel $W$ boson, respectively. However, the $t W$-channel overlaps at NLO with 
Table 1 Scale choices for all three single-top production channels

\begin{tabular}{lll}
\hline Process & Scales $\left(N_{f}=5\right)$ & Scales $\left(N_{f}=4\right)$ \\
\hline$p p \rightarrow t q / \bar{t} q$ (t-channel) & $-Q_{W}^{2}$ & $\mu_{\mathrm{F}}^{2}=\mu_{\mathrm{Q}}^{2}=m_{\mathrm{T}}^{t 2}, \mu_{\mathrm{R}}^{2}=m_{\mathrm{T}}^{b 2}$ \\
$p p \rightarrow t \bar{b} / \bar{t} b$ (s-channel) & $Q_{W}^{2}$ & $Q_{W}^{2}$ \\
$p p \rightarrow t W^{-} / \bar{t} W^{+}$(associated production) & $m_{\mathrm{T}}^{t 2}$ & - \\
\hline Here, $m_{\mathrm{T}}^{t 2}=m_{t}^{2}+p_{\mathrm{T}}^{t 2}$ and similar for the transverse mass of the $b$ quark, $m_{\mathrm{T}}^{b}$
\end{tabular}

$t \bar{t}$ production. ${ }^{1}$ This overlap is resolved using the diagram removal technique of [18], excluding doubly resonant diagrams. ${ }^{2}$ To assess uncertainties due to the flavour-scheme, we further calculate both the $t$ - and $s$-channel processes in the five- and four-flavour schemes. The $t W$-channel is calculated in the five-flavour scheme only, as the aforementioned ambiguous and gauge-dependent removal of resonant $t \bar{t}$ production is already present at LO in the four-flavour scheme $[35,36]$. The dominant background processes for the analysis in Sect. 4, $t \bar{t}$ production and $W$-boson production in association with at least one light- and one $b$-jet, also use the Mc@NLO technique.

Tree-level matrix elements and subtraction terms in the Catani-Seymour dipole formalism [38-40] are generated using the AMEGIC [41] and COMIX [42] matrixelement generators. One-loop matrix elements are taken from the OPENLOOPS library [43], relying on COLLIER [44], CUTTOOLs [45] and OnELOOP [46]. All partons are evolved from their high scales at production to low scales through a Catani-Seymour dipole shower, Css [47].

Top quarks are produced on-shell with $m_{t}=172.5 \mathrm{GeV}$ in the zero-width approximation, before they are decayed into a $W$ boson and a bottom quark. As in [48] the kinematics of the decay are adjusted a posteriori to the physical width of the top quark and the $W$ boson by redistributing their masses according to the respective Breit-Wigner distribution. These $W$ bosons are further decayed, either leptonically $(\ell=e, \mu, \tau)$, or semi-leptonically in the case of the $t \bar{t}$ background simulation. $W$ bosons that are not part of the top-quark decay chain, e.g. in the $t W$ channel, are decayed hadronically. The full decay chain accounts for spin-correlations and intermediate QCD and QED corrections through either constrained parton-shower evolution or soft-photon resummation in the YFS scheme $[49,50]$. The

\footnotetext{
${ }^{1}$ A fully consistent treatment of the $t W$-channel at NLO would involve the calculation of $W^{+} W^{-} b \bar{b}$ production, with the $t W$-channel simply being the singly-resonant contribution for observables that are inclusive in one of the $b$-quarks [33-37].

2 This naïve removal of all diagrams with resonant $\bar{t} / t$ propagators from the real emission corrections to $t W / \bar{t} W$ production is not gauge invariant. While such diagrams are present in both the $g g$ and the $q \bar{q}$ channels, but not the $g q$ and $g \bar{q}$ channels, only the $g g$ channel exhibits a gauge dependence due to its inclusion of diagrams with triple gluon vertices. This gauge dependence was studied explicitly in [18] and was found to be zero in covariant and negligible for non-covariant gauges.
}

Table 2 Scale choices for $W+$ jets and top-pair production

\begin{tabular}{ll}
\hline process & scales \\
\hline$p p \rightarrow W j b$ & $\frac{1}{4} H_{\mathrm{T}}^{\prime 2}$ \\
$p p \rightarrow t \bar{t}$ & $-\frac{1}{1 / \hat{s}+1 / \hat{t}+1 / \hat{u}}$ \\
\hline
\end{tabular}

Here, $H_{\mathrm{T}}^{\prime 2}=\sum_{i \in b, j} p_{\mathrm{T}, i}^{2}+m_{\mathrm{T}, \mathrm{W}}^{2}$. The scales for top-pair production are the default choice in the SHERPA event generator for the production of massive or massless QCD object in hadronic collisions

branching ratios are correctly taken into account throughout, with the exception of the total cross sections in Sect. 3 which are quoted for inclusive single-top production.

We further include the simulation of multiple parton interactions according to the method laid down in [51]. Its SHERPA implementation has been described in [52]. A hadronisation simulation [53] and hadron decays, including both hadronic and leptonic $\tau$ decays and supplemented by higher order QED corrections [50], are also included, in order to arrive at a particle-level calculation.

The hard interaction and its matching to the parton shower are characterised by three scales: the renormalisation scale $\mu_{\mathrm{R}}$, the factorisation scale $\mu_{\mathrm{F}}$, and the resummation scale $\mu_{\mathrm{Q}}$. The latter can be identified as the parton-shower starting scale. For the different single-top signal channels, the scales are set as listed in Table 1 . For the $t \bar{t}$ - and $W$-boson backgrounds, the scale choices are listed in Table 2. For $t \bar{t}$, the clustering algorithm of the MEPs@NLO multi-jet merging method [54-57] determines the emission scales up to the scale of the $2 \rightarrow 2$ core process. The PDFs for our central value are given by the NNPDF 3.0 set at NLO [58] in the appropriate flavour number scheme, interfaced through LHAPDF 6 [59]. The electroweak couplings $\alpha$ are evaluated with the $G_{\mu}$ scheme as suggested in [60]. The CKM matrix is set to the unit matrix, in particular $V_{t b}=1$. All other input parameters are detailed in Table 3.

Theory uncertainties are generated on-the-fly using the internal reweighting of SHERPA [61]. Where scale variations are given, they amount to the envelope over a 7-point scale variation, independently multiplying $\mu_{\mathrm{F}}$ and $\mu_{\mathrm{R}}$ by factors of two and one half, but not allowing variations where one scale is scaled up and the other one down. Where clustering is used in a calculation, only the core process scale is affected by the variation, the clustering scales are kept at their central values. To estimate PDF errors, the variations for the NNPDF 
Table 3 Numerical values of all input parameters

$$
\begin{aligned}
& G_{\mu}=1.16639 \cdot 10^{-5} \mathrm{GeV}^{2} \\
& m_{W}=80.385 \mathrm{GeV} \\
& m_{Z}=91.1876 \mathrm{GeV} \\
& m_{b}=4.75 \mathrm{GeV} \\
& m_{t}=172.5 \mathrm{GeV}
\end{aligned}
$$$$
\begin{aligned}
& \Gamma_{W}=2.085 \mathrm{GeV} \\
& \Gamma_{Z}=2.4952 \mathrm{GeV} \\
& \Gamma_{b}=0 \\
& \Gamma_{t}=1.47015 \mathrm{GeV}
\end{aligned}
$$

In calculations where a given particle is present as a final state its width is set to zero. The value listed above is then used in the redistribution of its kinematics in the generation of its factorised decay. The bottom-quark mass is only used in four-flavour scheme calculations

replicas are combined as a statistical sample [58]. To vary $\alpha_{\mathrm{s}}$, we generate results for PDF variations that are fitted using different input values for $\alpha_{\mathrm{s}}\left(m_{Z}\right)$. The central value for it is 0.118 , and the variations are 0.117 and 0.119 . The $\alpha_{\mathrm{s}}$ error is then given as the envelope over the three corresponding predictions. PDF and $\alpha_{\mathrm{s}}$ variations are not applied to the parton shower. All three sources of uncertainties, the scale, PDF and $\alpha_{\mathrm{s}}$ uncertainties, are either added linearly or given individually, if not specified otherwise.

For observables other than total inclusive cross sections, we use the Rivet framework [62] for the object definitions, observable projections and binnings.

\section{Total and fiducial cross sections and uncertainties}

In this section we compare our results, computed with the setup detailed in the previous section, with recent measurements at the $8 \mathrm{TeV}$ LHC [5-10]. We start by examining the inclusive total cross sections for both $t$ - and $s$-channel topand antitop-production as well as for associated $t W^{-}$and $\bar{t} W^{+}$production.

For the $t$ channel, we also calculate cross sections for a "fiducial" phase-space region. This region is defined by particle-level cuts and a particle reconstruction that emulate the analysis strategy and object definitions used for MonteCarlo samples in a recent experimental $t$-channel single-top study [10]. First, leptons are dressed with all photons within a radius $R=0.1$, and then are required to have $\left|\eta_{\ell}\right|<2.5$ and $p_{T}^{\ell}>25 \mathrm{GeV}$. Dressed leptons that do not originate from any hadron decay (either directly or via an intermediate $\tau$ lepton decay) are then considered to be tagged leptons. We require exactly one tagged lepton. In the setups we use this is guaranteed implicitly: Any tagged lepton $\ell$ is generated via $W \rightarrow \ell$ or $W \rightarrow \tau \rightarrow \ell$. We further require a missing transverse momentum, $p_{T}^{\text {miss }}$, of at least $30 \mathrm{GeV}$. Jets are defined by the anti- $k_{T}$-algorithm [63] with a radius parameter $R=0.4, p_{T}>30 \mathrm{GeV}$ and $|\eta|<4.5$. They are built from all visible particles except for dressed leptons. If one of the jets lies within $R=0.4$ around the tagged lepton, the event is vetoed. Jets are tagged as $b$-jets by associating a $b$-hadron with a ghost-matching method [64], and if their pseudo-rapidity is $|\eta|<2.5$. Exactly one $b$-jet is required. Events are rejected if $m_{b \ell}>160 \mathrm{GeV}$ to stay away from the off-shell regions. Additionally, it is required that there is exactly one light jet, i.e. one that is not tagged as a $b$-jet.

Our results for the total and fiducial cross sections are listed in Table 4. For an easy overview, they are also visualised in Fig. 1. We generally find good agreement between the data and our predictions in all three channels for both the five- and the four-flavour schemes. When comparing both kinds of calculations, however, a number of differences are found in the description of the $t$-channel process. These differences originate in the difference of contributing topologies already at leading order in the $N_{f}=4$ and $N_{f}=5$ scheme calculations. While this process proceeds through an initial state gluon splitting into an explicit $b \bar{b}$ pair with finite transverse momentum in the $N_{f}=4$ scheme, this splitting is absorbed into the PDF in the $N_{f}=5$ calculation which in turn operates with an initial state bottom quarks collinear to the beam axis while the other bottom quark from that splitting remains an unresolved part of the proton remnant. We find that the total uncertainty, both in the total as well as the fiducial $t$-channel cross section, is dominated by the perturbative scale uncertainties, and both the PDF and $\alpha_{\mathrm{s}}$ uncertainties are subdominant. In particular, the perturbative scale uncertainties are larger in the $N_{f}=4$ calculation than in the $N_{f}=5$ one. This larger variation is driven by the renormalisation scale and its comparatively low central scale choice motivated by an optimal description of the $g \rightarrow b \bar{b}$ splitting dynamics. All other uncertainties behave similarly in both calculations. The $s$-channel process, on the other hand, shows only a marginal sensitivity on the choice of scheme. This behaviour is expected since in both the four and five massless flavour schemes the same topologies contribute at leading and next-to-leading order. We therefore only show the $N_{f}=5$ result in Fig. 1. Lastly, the uncertainties for associated $t W$ production are again dominated by the perturbative scale uncertainties, with the PDF and $\alpha_{\mathrm{s}}$ uncertainties amounting to a half and a quarter of their size, respectively.

Further, we compare our $t$-channel computation for the fiducial phase-space region as defined above for the reconstructed top-quark transverse momentum and the light-jet rapidity $y_{j_{1}}$ with ATLAS data [10] in Fig. 2. To reconstruct the top quark momentum, we first reconstruct the $W$-boson momentum from the tagged (dressed) lepton momentum and $p_{T}^{\text {miss }}$, using $m_{W}$ as a constraint. The top quark is then reconstructed by adding the four-momenta of the reconstructed $W$ boson and the $b$-jet. Again, we can establish good agreement between our simulation and data.

In a next step, to further investigate the behaviour of our calculations and their associated uncertainties, we compare in Fig. 3 inclusive $t$ - and $s$-channel production with lep- 
Table 4 Total and fiducial single-top production cross sections in picobarn

\begin{tabular}{|c|c|c|c|c|c|c|c|c|c|c|c|c|}
\hline & \multicolumn{4}{|c|}{ SHERPA $\left(N_{f}=5\right)$} & \multicolumn{4}{|c|}{ SHERPA $\left(N_{f}=4\right)$} & \multicolumn{2}{|c|}{$\frac{\text { ATLAS }}{[7,8,10]}$} & \multicolumn{2}{|l|}{$\frac{\mathrm{CMS}}{[5,6,9]}$} \\
\hline & & $\mu_{R, F}$ & $\alpha_{\mathrm{s}}$ & PDF & & $\mu_{R, F}$ & $\alpha_{\mathrm{s}}$ & PDF & & tot. & & tot. \\
\hline \multicolumn{13}{|c|}{$t$-channel } \\
\hline \multicolumn{13}{|c|}{ tot. } \\
\hline$t$ & 58.3 & $\begin{array}{l}+1.8 \\
{ }_{-1.4}\end{array}$ & $\begin{array}{l}+0.4 \\
{ }_{-0.6}\end{array}$ & \pm 0.7 & 58.3 & $\begin{array}{l}+2.8 \\
-3.6\end{array}$ & $\stackrel{+}{+0.7}^{0.6}$ & \pm 0.6 & 56.7 & $\begin{array}{l}+4.3 \\
-3.8\end{array}$ & 53.8 & \pm 4.7 \\
\hline $\bar{t}$ & 32.1 & $\begin{array}{l}+1.0 \\
-0.8\end{array}$ & $\begin{array}{l}+0.3 \\
-0.4\end{array}$ & \pm 0.5 & 34.7 & $\begin{array}{l}+3.5 \\
-3.0\end{array}$ & ${ }_{-0.5}^{+0.5}$ & \pm 0.5 & 32.9 & $\begin{array}{l}+3.0 \\
-2.7\end{array}$ & 27.6 & \pm 4.0 \\
\hline \multicolumn{13}{|c|}{ fid. } \\
\hline$t$ & 9.30 & $\begin{array}{l}+0.36 \\
{ }_{-0.29}\end{array}$ & $\begin{array}{l}+0.06 \\
{ }_{-0.10}\end{array}$ & \pm 0.11 & 9.35 & $\begin{array}{l}+0.63 \\
{ }_{-0} .69\end{array}$ & $\begin{array}{l}+0.09 \\
{ }_{-0.11}\end{array}$ & \pm 0.10 & 9.78 & \pm 0.57 & - & \\
\hline $\bar{t}$ & 5.09 & $\begin{array}{l}+0.21 \\
-0.17\end{array}$ & $\begin{array}{l}{ }_{-0.06}^{+0.04} \\
-0.0\end{array}$ & \pm 0.08 & 5.72 & $\begin{array}{l}+0.71 \\
-0.57\end{array}$ & $\begin{array}{l}+0.08 \\
{ }_{-0.09}\end{array}$ & \pm 0.08 & 5.77 & \pm 0.45 & - & \\
\hline \multicolumn{13}{|c|}{$s$-ch. } \\
\hline \multicolumn{13}{|c|}{ tot. } \\
\hline$t$ & 3.31 & $\begin{array}{l}+0.09 \\
{ }_{-0.07}\end{array}$ & ${ }_{-0.02}^{+0.01}$ & \pm 0.06 & 3.26 & $\begin{array}{l}+0.09 \\
{ }_{-0} .07\end{array}$ & ${ }_{-0.02}^{+0.01}$ & \pm 0.06 & 4.8 & $\begin{array}{l}+1.8 \\
-1.6\end{array}$ & 13.4 & \pm 7.3 \\
\hline $\bar{t}$ & 1.89 & $\begin{array}{l}+0.05 \\
{ }_{-0.04}\end{array}$ & ${ }_{-0.01}^{+0.01}$ & \pm 0.04 & 1.87 & $\begin{array}{l}+0.05 \\
-0.04\end{array}$ & ${ }_{-0.01}^{+0.01}$ & \pm 0.04 & & & & \\
\hline \multicolumn{13}{|c|}{$t W$-ch. } \\
\hline \multicolumn{13}{|c|}{ tot. } \\
\hline$t$ & 12.3 & $\begin{array}{l}+0.8 \\
-0.7\end{array}$ & ${ }_{-0.2}^{+0.2}$ & \pm 0.4 & - & & & & 23.0 & $\begin{array}{l}+3.7 \\
-3.9\end{array}$ & 23.4 & \pm 5.4 \\
\hline $\bar{t}$ & 12.3 & $\begin{array}{l}+0.8 \\
-0.7\end{array}$ & $\begin{array}{l}+0.2 \\
-0.2\end{array}$ & \pm 0.4 & - & & & & & & & \\
\hline
\end{tabular}

The omitted statistical errors for the SHERPA results are at least an order of magnitude smaller than their scale uncertainties. All SHERPA results are generated at MC@NLO accuracy. The fiducial cross sections are defined by cuts that are as close as possible to the ones used in the recent experimental analysis they are compared against [10], cf. Sect. 3. The quoted experimentally measured values only give the total uncertainty. For the $s$ - and the $t W$-channel, experimental results give the sum over top and anti-top production
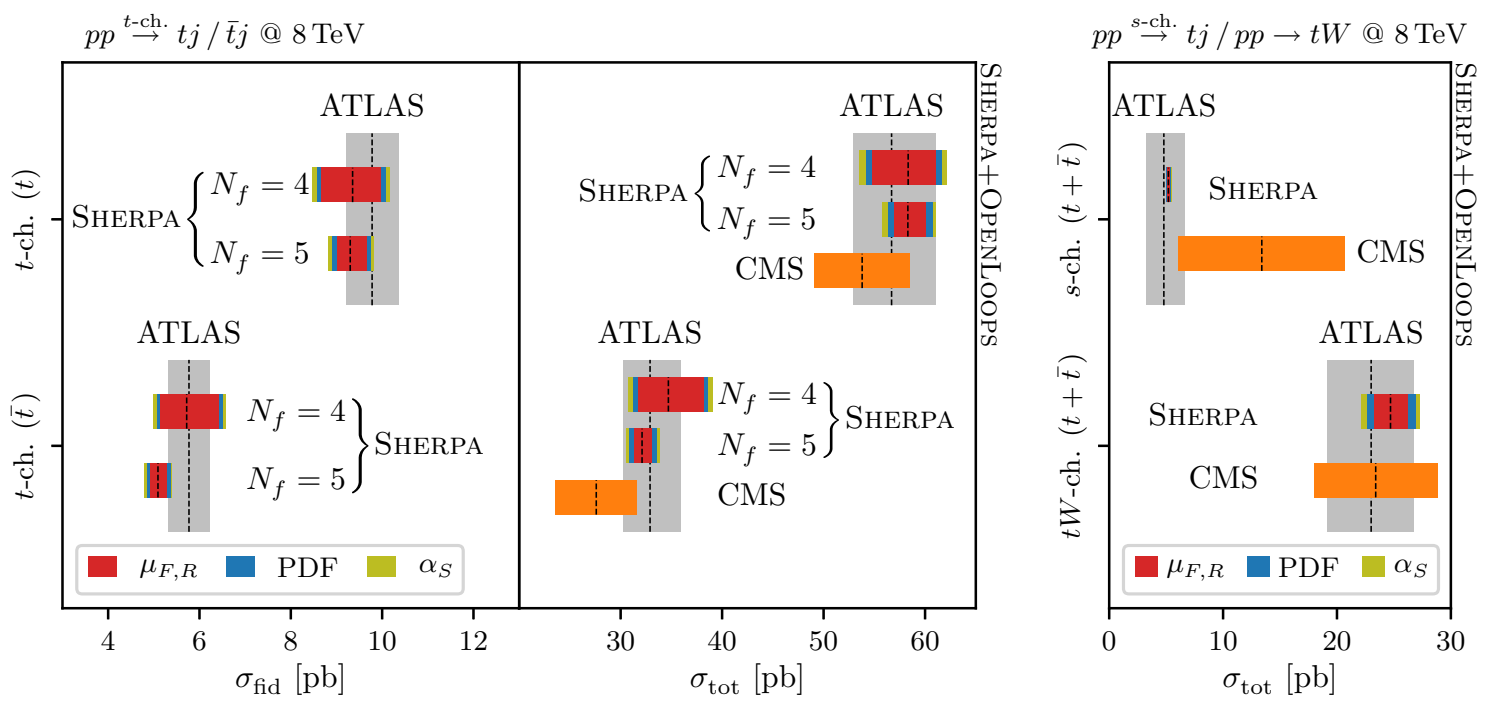

Fig. 1 Depiction of the cross sections from Table 4. The uncertainties for the SHERPA results are displayed staggered, i.e. the total width of the SHERPA band corresponds to the scale uncertainty (red), the PDF uncertainty (blue) and the $\alpha_{\mathrm{s}}$ uncertainty (yellow) added linearly. Only the $N_{f}=5$ SHERPA result is shown for the $s$-channel, because the $N_{f}=4$ result is nearly identical. All SHERPA results are calculated at MC@NLO. The fiducial phase space region for $\sigma_{\text {fid }}$ is defined in Sect. 3

and a minimum transverse momentum of $30 \mathrm{GeV}$ from all final-state particles except for leptons and photons that are within a radius of $R=0.1$ around leptons. The jets are sorted into light and $b$-tagged ones using the $b$-hadron association method described earlier. The light jets are ordered by 


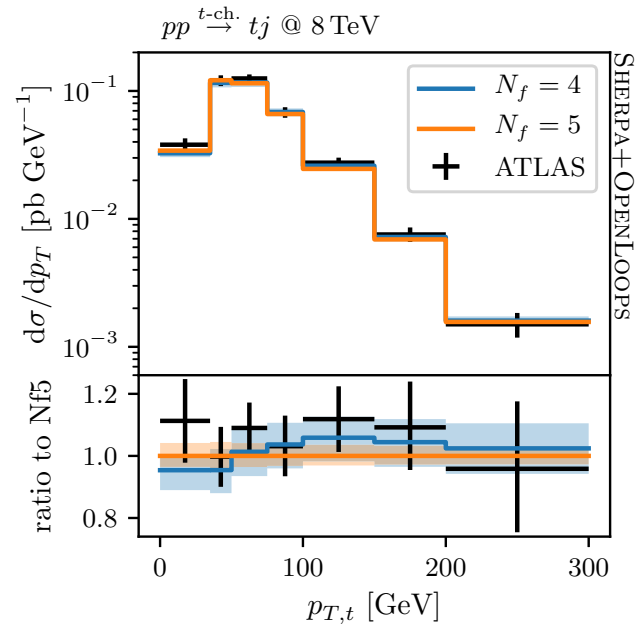

Fig. 2 Comparison of SHERPA MC@NLO predictions with ATLAS data [10] for the top-quark transverse momentum $p_{T, t}$ and the light-jet rapidity $y_{j_{1}}$ in $t$-channel single-top production. The SHERPA uncertainty

their transverse momentum, such that $j_{1}$ denotes the hardest or leading jet. We separately detail the uncertainties stemming from scale variations, the parton distributions, and the value of the strong coupling for top and anti-top production in both channels. For $t$-channel production, they are contrasted with the difference of the five- and four-flavour schemes, which we mostly find to be in good agreement. The four-flavour scheme results are not shown for the $s$ channel, because here both schemes lead to almost identical results, probably because the $s$-channel neither depends on $\alpha_{\mathrm{S}}$ nor the bottom PDF at LO. The top and anti-top quark in the $t$ channel are produced centrally, and the uncertainties are dominated by the renormalisation and factorisation scale variations. PDF uncertainties only become relevant beyond rapidities of $\left|y_{t}\right|>2$, which have little relevance in the $8 \mathrm{TeV}$ measurements. For top production the five- and four-flavour calculations agree on the level of a few percent throughout the entire rapidity range. The deviations are larger for anti-top production, in this case the scale uncertainties barely cover the difference. The production via $s$ channel is less central, but otherwise exhibits a very similar structure with respect to the uncertainties. Slightly larger differences can be observed in the leading light-jet rapidity $y_{j_{1}}$. It is produced predominantly at large rapidities in $t$-channel production, while it is produced centrally in the $s$ channel. The differences between the five- and four-flavour schemes are larger than for $y_{t}$. Also, they are again even more pronounced for anti-top production. The last quantity we assess is the leading-jet transverse momentum $p_{T, j_{1}}$. As the $p_{T}$ increases in $t$-channel production, the uncertainties become dominated by the PDF uncertainties. Conversely, in $s$-channel production the scale uncertainty rapidly increases as the $p_{T}$ increases, dominating the total uncertainty budget.

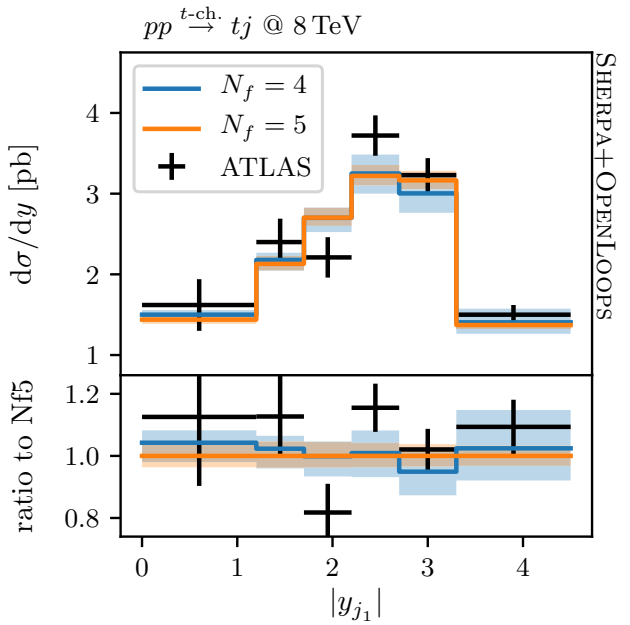

consists of the statistical, the $\alpha_{\mathrm{s}}$, the PDF and the (dominating) scale uncertainty, all added in quadrature. The distributions are calculated in the fiducial phase-space region as defined in Sect. 3

The difference between five-flavour scheme and four-flavour scheme predictions is most pronounced in this observable and increases to $10 \%$ at $p_{T}=200 \mathrm{GeV}$ for $t$-channel top productions and $40 \%$ for anti-top production. The difference in the flavour-scheme uncertainty originates in the different interplay of the up(down)-quark and (anti)bottom-quark PDF in the dominant five-flavour (anti)top quark production channel, and the up(down)-quark and the gluon PDF in the dominant four-flavour production channel, especially in their evolution to higher $Q^{2}$ probes.

This highlights that for a successful extraction of $\left|V_{t b}\right|$ from single-top production a good understanding of the bottom-quark PDF and its uncertainty is required. ${ }^{3}$ Whereas errors internal to a PDF set are usually taken into account for such a measurement [10], the spread over different PDF sets should also be included, as is done in [6]. Instead of extracting $\left|V_{t b}\right|$, single-top production cross sections can also be used to fit the bottom-quark PDF, assuming $\left|V_{t b}\right| \approx 1$. To explore this, we study both total and differential cross sections for $t$-channel single-top production varying the input PDF set, comparing central values of NNPDF 3.0, CT14 [67], MMHT2014 [68] and abm11 [67], all at NLO. To capture correlation effects we vary either all parton densities, or the bottom-quark density only while leaving the other densities at their default NNPDF 3.0 values.

Figure 4 shows the leading-jet rapidity $j_{y_{1}}$ (left column) and its transverse momentum $p_{T, j_{1}}$ (right column), in the fiducial region. In the top row, the PDF set is varied for all flavours, whereas in the bottom row, only the bottom and anti-bottom PDF is varied. In summary, when varying

\footnotetext{
${ }^{3}$ See [66] for a discussion of the light-quark PDF dependence of $t$ channel single top-quark production.
} 

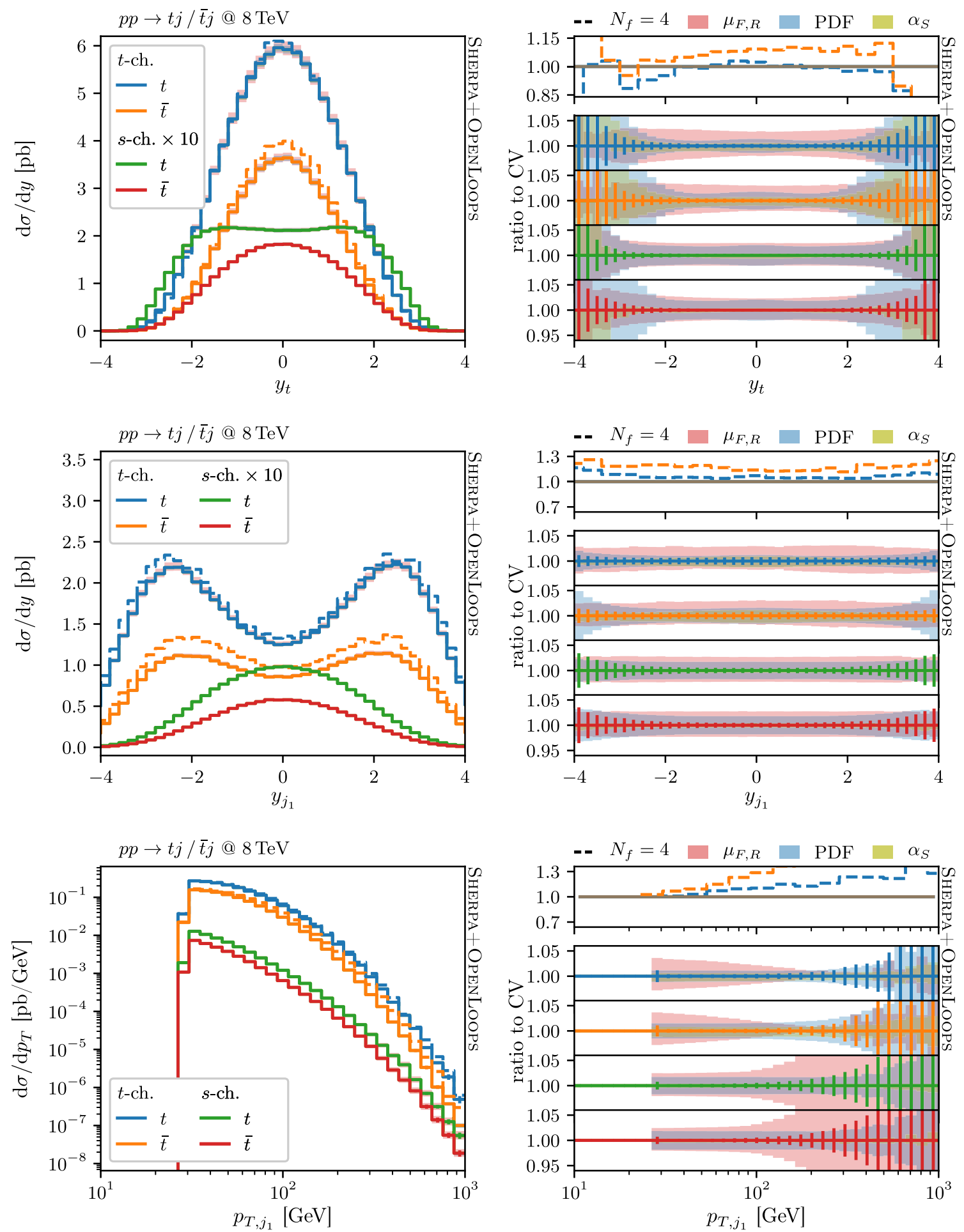

Fig. 3 The plots in the left panel show the distribution of the topand antitop-quark rapidity $y_{t}$ (top) as well as the leading-jet rapidity $y_{j_{1}}$ (centre) and its transverse momentum $p_{T, j_{1}}$ (bottom) for leptonic $t$ - and $s$-channel single-top production in the five-flavour scheme. No additional phase-space cuts apply. The plots in the right panel detail their respective uncertainties stemming from the choice for the scales (red band), parton distributions (blue band) and the value of $\alpha_{\mathrm{s}}$ (yellow band). Each such uncertainty budget is shown separately for each chan-

nel: $t$-channel top (blue) and anti-top (orange), and $s$-channel top (green) and anti-top (red) production. The additional panel at the top of each uncertainty breakdown shows the ratio of the four-flavour scheme calculation (dashed) to the corresponding five-flavour calculation (solid) for $t$-channel production. Note that the $s$-channel rapidity distributions have been scaled by a factor of ten. Also note the varying $y$ ranges of the flavour-scheme ratio panels 

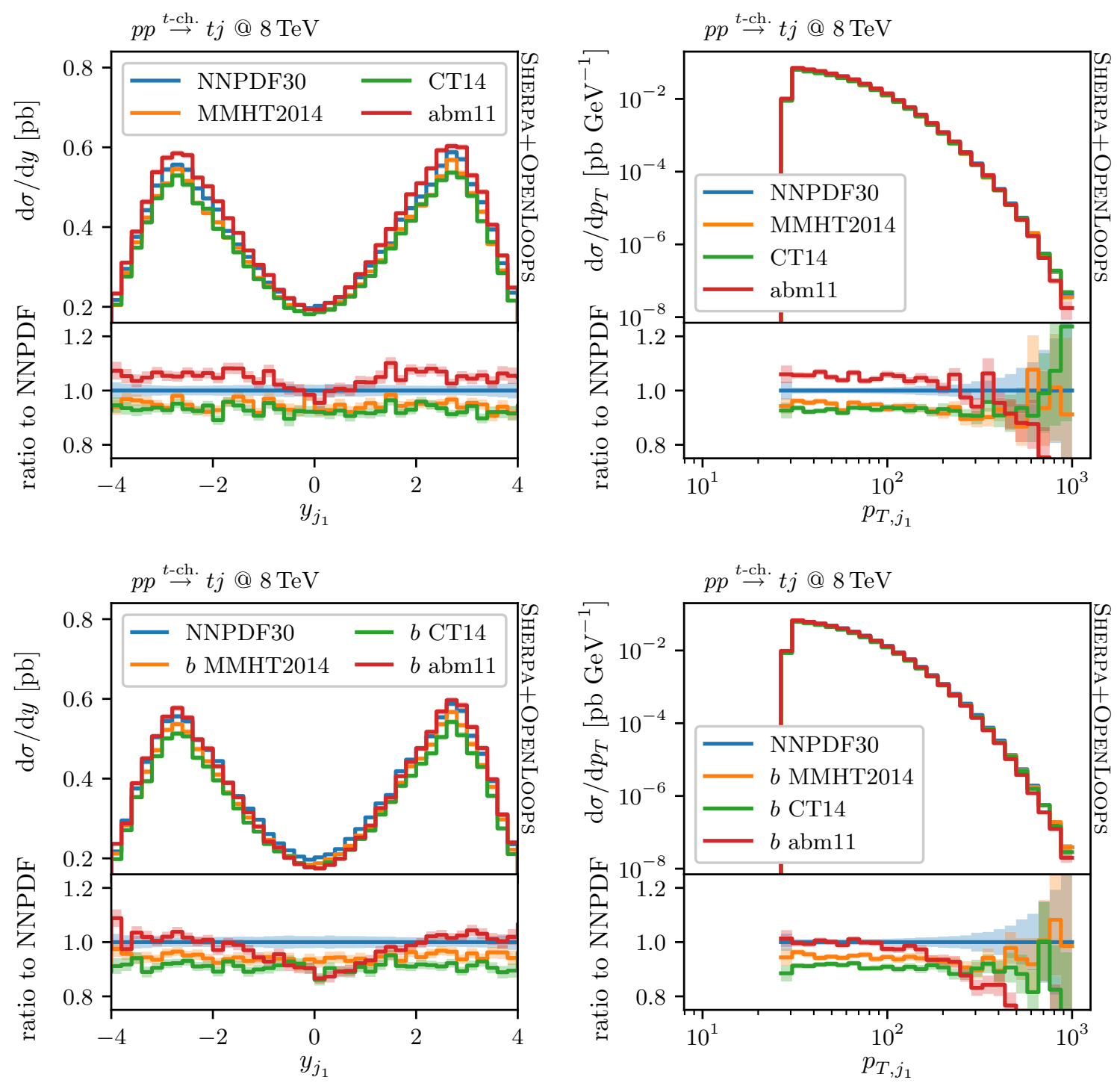

Fig. 4 The impact of different PDF sets on the leading-jet rapidity (left column) and transverse momentum (right column) in MC@NLO $t$ channel single-top production within the fiducial phase-space as defined

in Sect. 3. We show results for the variation of all PDFs (top row) and of the bottom PDF only (bottom row). The uncertainty band gives the statistical errors

among the NNPDF 3.0, the CT14 and the MMHT2014 sets, we find a mostly flat ratio between the rates and practically all relevant distributions, with CT14 and MMHT2014 approximately 5\% below NNPDF 3.0. This finding does not change much when only varying the bottom PDF, suggesting that the normalisation is driven by the respective bottom-quark densities, with the lighter quark and gluon densities agreeing among the PDF sets in the relevant phase-space regions. This is consistent with the ratios between the bottom PDFs in Fig. 5, where they are shown at the scale $Q=300 \mathrm{GeV}$. This is approximately the average factorisation scale for our $t$-channel single-top production. The distribution of the longitudinal momentum fraction peaks at $x \approx 5 \cdot 10^{-3}$, with an average of $x \approx 10^{-1}$. In this region we indeed find the

MMHT2014 and CT14 bottom PDF values to be 5-10\% smaller than the NNPDF 3.0 ones. For abm11, we observe in Fig. 4 for top production a normalisation offset with respect to NNPDF 3.0 of about $+5 \%$, which however completely vanishes when only varying the bottom-quark density. In addition, we find shape dependences at the level of $10 \%$ for the leading-jet rapidity distribution $y_{j_{1}}$. Similarly, the leading jet transverse momentum exhibits strongly divergent shapes of a similar magnitude as the five- to four-flavour calculation difference beyond $p_{T}>200 \mathrm{GeV}$.

These findings suggest that in order to improve the bottomquark distribution from single-top production, its measurement at higher luminosities and/or energies is mandatory such that both the high- $p_{T}$ and central rapidity regions 


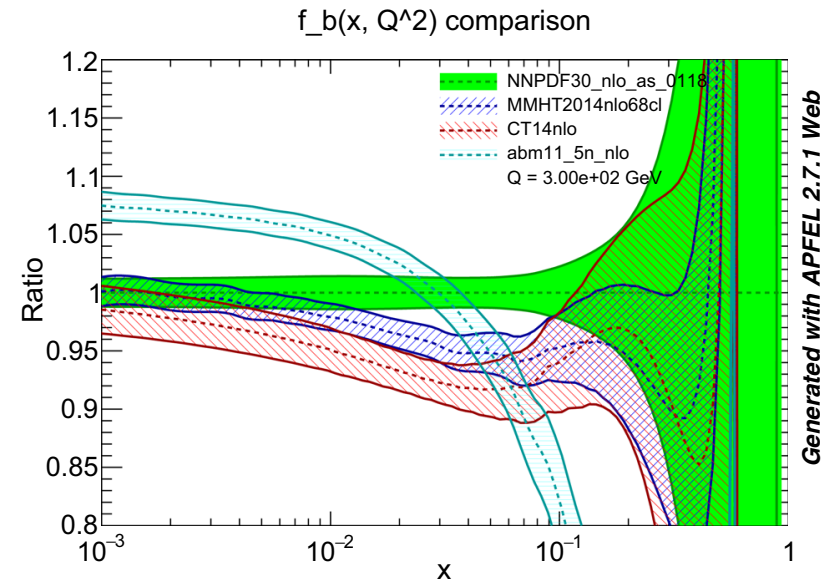

Fig. 5 Ratios between different PDF sets for the bottom PDF $f_{b}\left(x, Q^{2}\right)$, with the scale $Q$ set to the average factorisation scale for $t$-channel single-top production. The plot has been generated using the APFEL library [65]

can be explored with competitive statistical uncertainties. In turn, this implies, despite the observed differences, that the bottom-quark PDF uncertainty is sufficiently well understood for $\left|V_{t b}\right|$ extractions from single-top production with the $8 \mathrm{TeV}$ data.

\section{Signal-over-background ratio for different light-jet cuts}

Finally, we investigate the impact and effectiveness of different particle-level cuts to enhance the $t$-channel single-top signal over the background, consisting of $W$-boson and $t \bar{t}+$ jets production.

For this study we again use the fiducial phase-space region and object definitions as defined in Sect. 3, in accordance with the experimental analysis in [10]. However, aiming to further reduce the background, we study various alternatives to the requirement that there is exactly one light jet, $N_{1 \text {-jets }}=1$, and assess the effectiveness of these alternate cuts.

Focusing on the dominant $t$-channel production mode it is worthwhile to contemplate its kinematics. It is defined by the exchange of a colourless $W$ boson in the $t$-channel, giving rise to a light "tag" jet and the top quark which decays into a bottom-quark and a $W$ boson, with the latter subsequently decaying either into a lepton-neutrino or a quark-anti-quark pair. The colourless $t$-channel exchange suggests a kinematic similarity with weak-boson fusion events, with one light-quark current connected to a heavy-quark currentthe transition from bottom- to top-quark at Born level. This analogy implies that, while the top quark and its decay system remain nearly inert in the central region of the detector, the light tag jet is peaked in the forward regions, at rapidities of about or above $\left|y_{j}\right| \approx 2$, cf. Fig. 3. The contribution of the up-quark to the total top-quark production is larger than that of the down-quark to the total antitop-quark production. Through its valence bump at comparably large momentum fractions of $x \approx 0.15$ the mean rapidity of the tag jet will be somewhat larger for top than for anti-top production. Indeed, we find $\left\langle\left|y_{t}\right|\right\rangle=2.23$ and $\left\langle\left|y_{\bar{t}}\right|\right\rangle=2.03$ for top-quark and antitop-quark production, respectively, when using $N_{\text {l-jets }}=1$ as the light-jet multiplicity cut. This gives a difference of $\Delta\langle|y|\rangle=0.20$, a value that varies between $0.15 \ldots 0.22$ with the other cuts given below.

Due to the coherence property of QCD, additional radiation off the light quark line will typically also be quite forward, while radiation off the top quark is massively reduced due to the shielding of the collinear singularity by its mass. Therefore, additional QCD radiation in the central region will be depleted and mainly driven by secondary emissions from the top decay. This feature, depletion of radiation in the central region and a "rapidity gap" between the reconstructed top and the tag jet are absent in the backgrounds, which are not driven by colourless $t$-channel exchanges, but are more or less exclusively driven by the strong interaction between the two protons. This opens up possibilities for substantial improvements of the signal-to-background ratio through cuts on additional central hadronic or jet activity. In the following we test the effect of applying five different vetoes on central QCD radiation:

(a) a simple cut on the rapidity difference between the reconstructed top and any light-jet $j$,

$\Delta y_{t j}=\left|y_{t}-y_{j}\right|>y_{\text {cut }}$

(b) a cut on light-jet activity in the central region, by demanding

$G_{T}^{(0)}=\sum_{j \in \text { jets }}\left|p_{\perp, j}\right| \exp \left(-\left|y_{j}\right|\right)<G_{T, \text { cut }}^{(0)}$,

(c) a cut on light-jet activity in the region around the top, by demanding

$G_{T}^{(t)}=\sum_{j \in \text { jets }}\left|p_{\perp, j}\right| \exp \left(-\left|y_{j}-y_{t}\right|\right)>G_{T, \text { cut }}^{(t)}$,

(d) a cut on the hadronic activity in the central region, by demanding

$g_{T}^{(0)}=\sum_{i \in \text { tracks }}\left|p_{\perp, i}\right| \exp \left(-\left|y_{i}\right|\right)<g_{T, \text { cut }}^{(0)}$,

(e) a cut on the hadronic activity in the region around the top, by demanding 


$$
g_{T}^{(t)}=\sum_{i \in \text { tracks }}\left|p_{\perp, i}\right| \exp \left(-\left|y_{i}-y_{t}\right|\right)>g_{T, \text { cut }}^{(t)} .
$$

Here, we use the properties of charged tracks to characterise the hadronic activity. They are defined to have $|\eta|<2.5$ and $p_{T}>400 \mathrm{MeV}$ and we discard tracks that are within $R=0.4$ around the $b$-jet or within $R=0.1$ around the lepton. In consequence, no jet or track from the reconstructed top enters the sum in the definition of the measures (b)-(e). While all five options enhance the contribution from topologies that exhibit rapidity gaps, they vary in their restrictiveness. Only option (a) rejects all configurations for which the leading jet and the top-quark are too close in rapidity. In contrast, the other four options weigh the occurring radiation by their distance either from the centre of the detector, $G_{T}^{(0)}$ and $g_{T}^{(0)}$, or the reconstructed top-quark, $G_{T}^{(t)}$ and $g_{T}^{(t)}$. Options (b) and (d) therefore do not necessarily lead to a rapidity gap between the top-quark and the light jet, but instead to a gradual depletion of the hadronic activity in the central detector. It is worth stressing that vetoes on jets with a transverse momentum smaller than about $20 \mathrm{GeV}$ are very ambitious and riddled with large theoretical and experimental uncertainties. This is certainly also true for vetoes on overall hadronic activity as manifest in $g_{T}^{(0, t)}$ which is driven by non-perturbative effects such as the underlying event or hadronisation. At the same time, this may be an avenue for further study, capitalising on recent advances in our understanding of QCD interactions.

The signal-over-background $(S / B)$ ratios for all five versions of inducing a rapidity gap and the remaining signal cross sections are shown in Fig. 6. While $S / B$ ratios of 4 and higher can be achieved, they of course come at the cost of a vanishingly small signal cross section. The best results can be obtained by restricting central-jet or hadronic activity. While the $S$ / $B$ ratios are similar between the two approaches, the jet-based veto removes less signal cross section than the track-based one and is therefore preferable. Interestingly, demanding a depleted central detector achieves at least as good and in most cases better results, than a depletion in a rapidity region relative to the reconstructed top quark. This is true both in terms of $S / B$ ratios and of the remaining signal cross section. Of course, since the top-quark itself is predominantly produced very centrally, the differences are moderate. The track-based rejections fare very similarly to the jet-based rejections for large rejection scales, but are more repressive at small rejection scales. While backgrounds are suppressed very well, also the signal cross section is lost. Good compromises are offered by central-jet or track veto scales of around $5-10 \mathrm{GeV}$ or rapidity gaps of 2.5 units.

To examine the effect of the above rapidity gap inducing phase space restrictions on the leading light-jet rapidity, we define four sets of cuts:

1. $G_{T \text {, cut }}^{(0)}=10 \mathrm{GeV}$,
2. $G_{T, \text { cut }}^{(t)}=10 \mathrm{GeV}$,
3. $g_{T, \text { cut }}^{(0)}=5 \mathrm{GeV}$,
4. $g_{T, \text { cut }}^{(t)}=5 \mathrm{GeV}$.

The resulting distributions are shown in Fig. 7. The upper panel shows the leading light-jet rapidity distributions for $t$ channel top and anti-top production after the application of (1) or (2) and contrast them with the distributions after the application of the original $N_{1 \text {-jets }}=1$ or $y_{\text {cut }}=2.5$ restrictions. They are accompanied by the corresponding distributions of the $t \bar{t}$ and $W j b$ background processes. The lower panel shows the same distributions, now applying the restrictions of (3) and (4) instead.

Because the $N_{\text {-jets }}=1$ requirement does not enforce a rapidity gap, we find that when using this requirement a large number of background events survive where the signal cross section is minimal. Among the three other options, $G_{T, \text { cut }}^{(t)}=10 \mathrm{GeV}$ and $y_{\text {cut }}=2.5$ largely give very similar results, with minor difference in the central region for the $W j b$ background. $G_{T \text {,cut }}^{(0)}=10 \mathrm{GeV}$ induces a more aggressively depleted central detector, but leaves an increased signal rate at $\left|y_{j_{1}}\right| \approx 2.5$, accumulating to a larger signal cross section throughout the spectrum. Similar rapidity-dependent depletions are induced in the background processes. However, as the bulk of their cross section resides at central rapidities, their rates are reduced much more strongly. Decreasing alternatively the track-veto scale in the central region or the vicinity of the top-quark, as detailed in the lower panel of Fig. 7, reduces the background rates to negligible values, but also has an adverse effect on the signal cross section, as we have already observed before. Only small signal regions beyond $\left|y_{j_{1}}\right| \gtrsim 2.5$ survive.

Turning the above line-of-thought around, requiring a minimal remaining signal cross-section after cuts of $1 \mathrm{pb}$, the best value for $S / B$ using a plain rapidity gap requirement is about 2.5 when using $y_{\text {cut }}=2.5$. While the light-jet suppression in the top vicinity achieves similar results, leaving a signal cross section of 1 pb with a $S / B$ of about 2.4 with $G_{T \text {,cut }}^{(t)}=3.5 \mathrm{GeV}$, the top-independent central jet veto performs best, reaching a $S / B$ ratio of approximately 4 at a signal cross section of $1 \mathrm{pb}$ with $G_{T \text {, cut }}^{(0)}=3 \mathrm{GeV}$.

\section{Summary}

We reported on the simulation of single top-quark production in the $s-, t$ - and $t W$-channels with the SHERPA event generator at MC@NLO accuracy. After validating our results with experimental data for various cross sections and through selected differential observables, we focused on two short phenomenological studies. First, we analysed the impact of the bottom PDF on various observables. We find that for most 

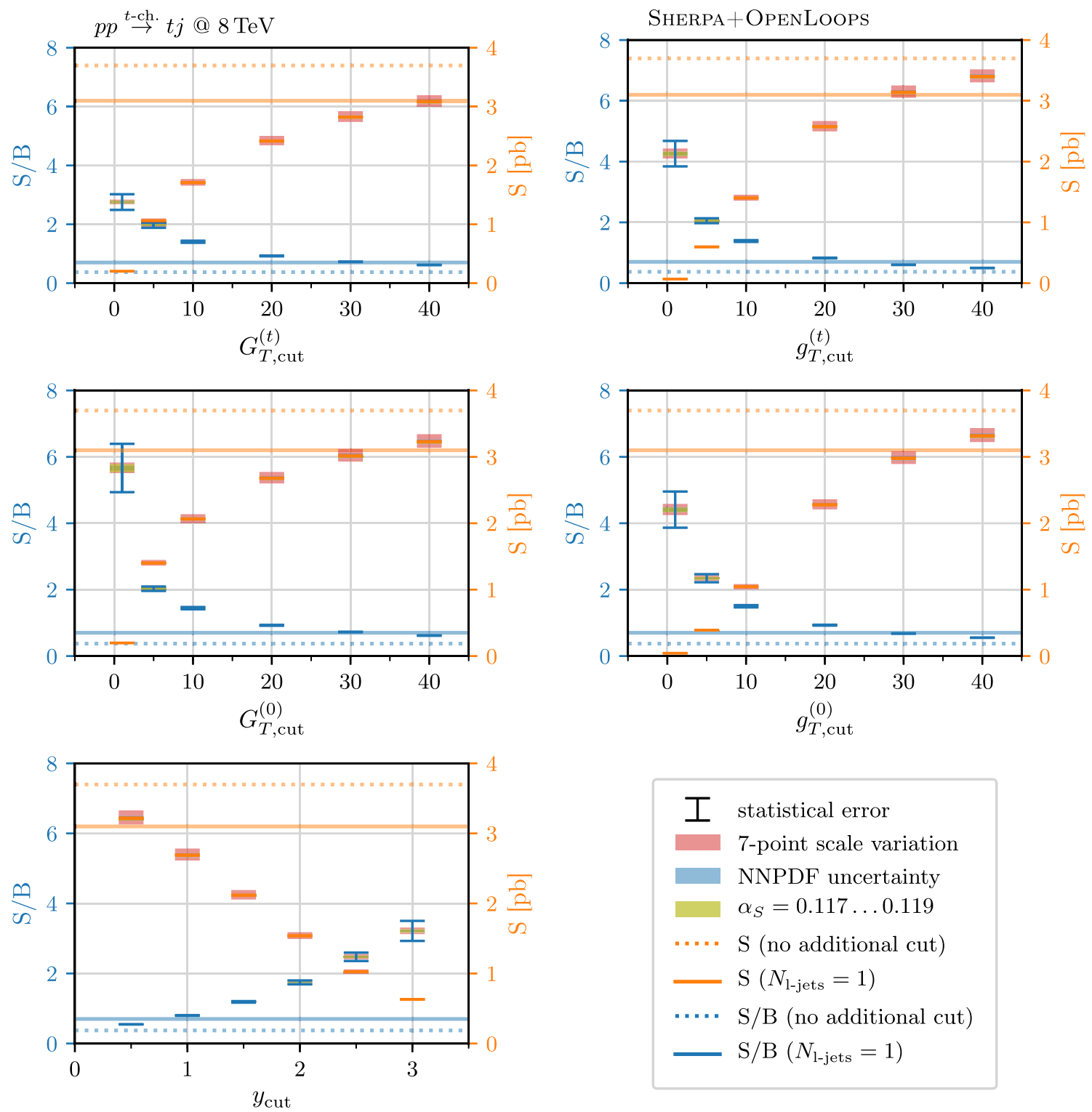

Fig. 6 Signal-over-background ratios for different cut alternatives to the $N_{\text {l-jets }}=1$ requirement in the fiducial phase space definition as described in Sect. 3. The signal is $t$-channel single-top production, the background consists of the sum over $t \bar{t}$ and $W j b$ production. Scale uncertainties are included only for the signal in the $S / B$ ratios. PDF

of the standard PDFs the shapes are very robust, on the level of $5 \%$ or below, and that the main differences are in the total normalisation, i.e. the overall cross section with bottom PDF induced uncertainties of up to about $10 \%$. The only exception is the abm11 PDF set, which also shows some shape distortions. Overall, this provides ample motivation to use precision determinations of single top-quark production as a means to directly measure the absolute value of the CKM element $\left|V_{t b}\right|$. Second, we focused on the $t$-channel production mode and applied a variety of vetoes on QCD radia- tion in central rapidity regions. To this end we introduced a number of observables, essentially scalar sums of transverse momenta of jets or charged tracks, weighted with an exponential form suppressing them at large rapidities or rapidity differences with respect to the top-quark system. As they exploit the topological differences of the signal and its background processes, it is unsurprising that all five versions of such an additional requirement provided significant enhancements of the $S / B$ ratio of around 2-4 while simultaneously keeping the signal cross section at $1 \mathrm{pb}$ or above. This leads 

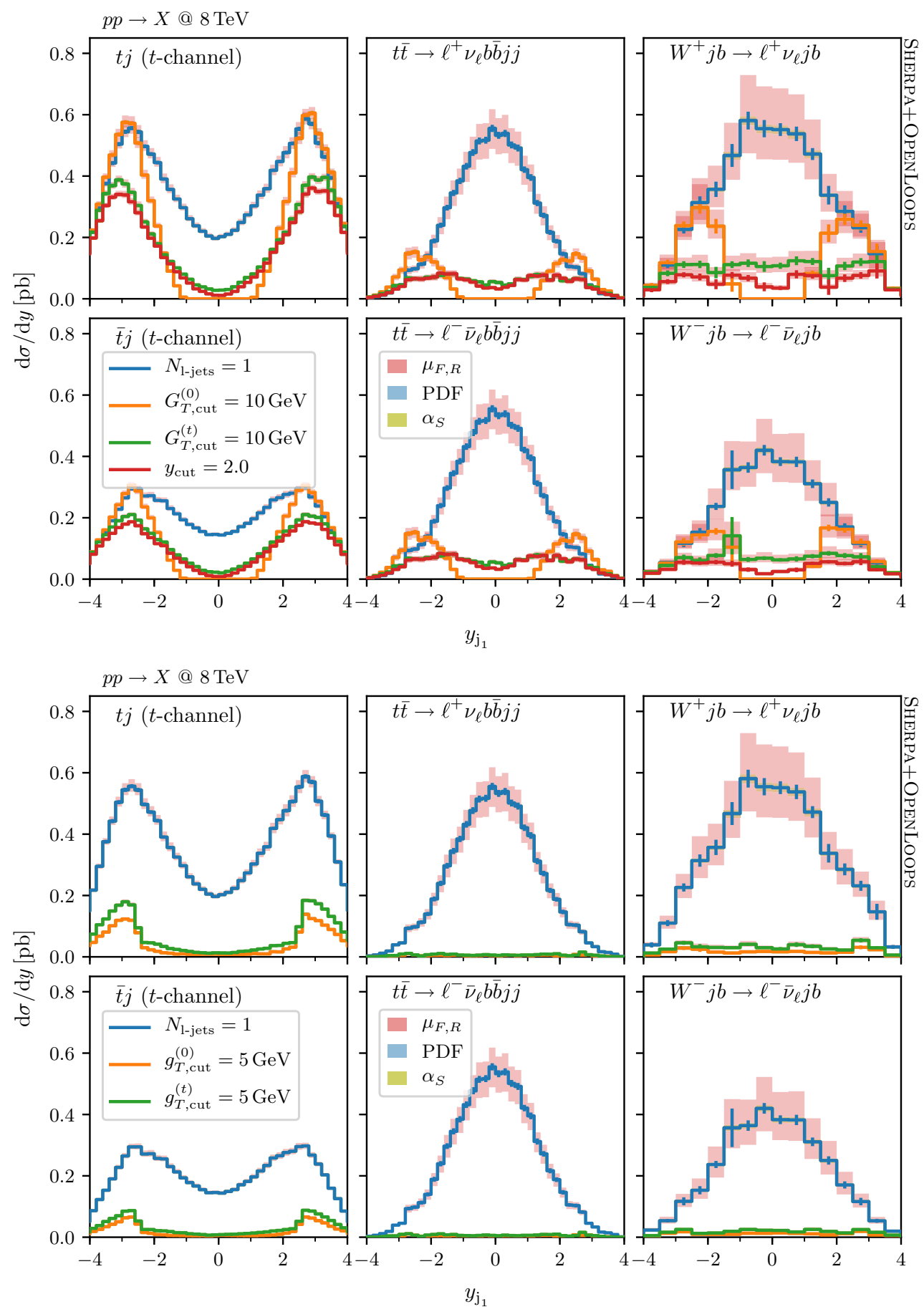

Fig. 7 SHERPA MC@NLO results for the leading-jet rapidity $y_{j_{1}}$ for different signal channels and background processes in the fiducial phase space defined in Sect. 3, with various alternate cuts replacing the $N_{\text {l-jets }}=1$ requirement

us to suggest to replace the flat restriction on light-jet activity used so far in experimental analysis by any of the rapidity-gap inducing candidates suggested in this paper and investigate their behaviour further in subsequent experimental studies.

Acknowledgements This work was supported by the European Union as part of the EU Marie Curie Research Training Network MCnet
(MRTN-CT-2006-035606). We thank all members of the SHERPA collaboration for valuable input and S. Höche in particular for providing help with the COMIX matrix element generator in algorithmically differentiating the three different single-top processes in NLO calculations.

Open Access This article is distributed under the terms of the Creative Commons Attribution 4.0 International License (http://creativecomm ons.org/licenses/by/4.0/), which permits unrestricted use, distribution, 
and reproduction in any medium, provided you give appropriate credit to the original author(s) and the source, provide a link to the Creative Commons license, and indicate if changes were made.

Funded by SCOAP ${ }^{3}$.

\section{References}

1. T.M.P. Tait, C.P. Yuan, Single top quark production as a window to physics beyond the standard model. Phys. Rev. D63, 014018 (2000). arXiv:hep-ph/0007298 [hep-ph]

2. J.A. Aguilar-Saavedra, Single top quark production at LHC with anomalous Wtb couplings. Nucl. Phys. B 804, 160-192 (2008). arXiv:0803.3810 [hep-ph]

3. J. Gao, C.S. Li, L.L. Yang, H. Zhang, Search for anomalous top quark production at the early LHC. Phys. Rev. Lett. 107, 092002 (2011). arXiv:1104.4945 [hep-ph]

4. G. Aad et al., The ATLAS collaboration, Measurement of the $t$ channel single top-quark production cross section in $p p$ collisions at $\sqrt{s}=7 \mathrm{TeV}$ with the ATLAS detector. Phys. Lett. B 717, 330 350 (2012). arXiv:1205.3130 [hep-ex]

5. S. Chatrchyan et al., The CMS collaboration, Observation of the associated production of a single top quark and a $W$ boson in $p p$ collisions at $\sqrt{s}=8 \mathrm{TeV}$, Phys. Rev. Lett. 112(23), 231802 (2014). arXiv:1401.2942 [hep-ex]

6. V. Khachatryan et al., The CMS collaboration, Measurement of the t-channel single-top-quark production cross section and of the $\left|V_{t b}\right| \mathrm{CKM}$ matrix element in pp collisions at $\sqrt{s}=8 \mathrm{TeV}$. JHEP 06, 090 (2014). arXiv:1403.7366 [hep-ex]

7. G. Aad et al., The ATLAS collaboration. Measurement of the production cross-section of a single top quark in association with a $W$ boson at $8 \mathrm{TeV}$ with the ATLAS experiment. JHEP 01, 064 (2016). arXiv: 1510.03752 [hep-ex]

8. G. Aad et al., The ATLAS collaboration. Evidence for single topquark production in the $s$-channel in proton-proton collisions at $\sqrt{s}=8 \mathrm{TeV}$ with the ATLAS detector using the matrix element method. Phys. Lett. B 756, 228-246 (2016). arXiv:1511.05980 [hep-ex]

9. V. Khachatryan et al., The CMS collaboration, Search for s channel single top quark production in pp collisions at $\sqrt{s}=7$ and $8 \mathrm{TeV}$. JHEP 09, 027 (2016). arXiv:1603.02555 [hep-ex]

10. M. Aaboud et al., The ATLAS collaboration, Fiducial, total and differential cross-section measurements of $t$-channel single topquark production in $p p$ collisions at $8 \mathrm{TeV}$ using data collected by the ATLAS detector, Eur. Phys. J. C77(8), 531 (2017). arXiv:1702.02859 [hep-ex]

11. J. Alwall, R. Frederix, J .M. Gerard, A. Giammanco, M. Herquet, S. Kalinin, E. Kou, V. Lemaitre, F. Maltoni, Is $V_{t b} \simeq 1$ ? Eur. Phys. J. C49, 791-801 (2007). arXiv:hep-ph/0607115 [hep-ph]

12. T. Aaltonen et al., The CDF collaboration, Observation of single top quark production and measurement of $|\mathrm{Vtb}|$ with CDF. Phys. Rev. D 82, 112005 (2010). arXiv:1004.1181 [hep-ex]

13. M .C. Smith, S. Willenbrock, QCD and Yukawa corrections to single top quark production via $q \bar{q} \rightarrow t \bar{b}$. Phys. Rev. D54, 66966702 (1996). arXiv:hep-ph/9604223 [hep-ph]

14. B.W. Harris, E. Laenen, L. Phaf, Z. Sullivan, S. Weinzierl, The fully differential single top quark cross-section in next to leading order QCD. Phys. Rev. D 66, 054024 (2002). arXiv:hep-ph/0207055 [hep-ph]

15. G. Bordes, B. van Eijk, Calculating QCD corrections to single top production in hadronic interactions. Nucl. Phys. B435, 23-58 (1995)

16. W .T. Giele, S. Keller, E. Laenen, QCD corrections to $W$ boson plus heavy quark production at the Tevatron. Phys. Lett. B372, 141-149 (1996). arXiv:hep-ph/9511449 [hep-ph]
17. S. Frixione, E. Laenen, P. Motylinski, B.R. Webber, Singletop production in MC@NLO. JHEP 03, 092 (2006). arXiv:hep-ph/0512250 [hep-ph]

18. S. Frixione, E. Laenen, P. Motylinski, B.R. Webber, C.D. White, Single-top hadroproduction in association with a $\mathrm{W}$ boson. JHEP 07, 029 (2008). arXiv:0805.3067 [hep-ph]

19. S. Alioli, P. Nason, C. Oleari, E. Re, NLO single-top production matched with shower in POWHEG: $s$ - and $t$-channel contributions. JHEP 09, 111 (2009). arXiv:0907.4076 [hep-ph]

20. E. Re, Single-top Wt-channel production matched with parton showers using the POWHEG method. Eur. Phys. J. C71, 1547 (2011). arXiv:1009.2450 [hep-ph]

21. J.M. Campbell, R. Frederix, F. Maltoni, F. Tramontano, Nextto-leading-order predictions for t-channel single-top production at hadron colliders. Phys. Rev. Lett. 102, 182003 (2009). arXiv:0903.0005 [hep-ph]

22. R. Frederix, E. Re, P. Torrielli, Single-top t-channel hadroproduction in the four-flavour scheme with POWHEG and aMC@NLO. JHEP 09, 130 (2012). arXiv:1207.5391 [hep-ph]

23. J.M. Campbell, R.K. Ellis, F. Tramontano, Single top production and decay at next-to-leading order. Phys. Rev. D70, 094012 (2004). arXiv:hep-ph/0408158 [hep-ph]

24. M. Brucherseifer, F. Caola, K. Melnikov, On the NNLO QCD corrections to single-top production at the LHC. Phys. Lett. B736, 58-63 (2014). arXiv:1404.7116 [hep-ph]

25. E.L. Berger, J. Gao, C .P. Yuan, H .X. Zhu, NNLO QCD corrections to t-channel single top-quark production and decay. Phys. Rev. D94(7), 071501 (2016). arXiv:1606.08463 [hep-ph]

26. E. L. Berger, J. Gao, H. X. Zhu, Differential distributions for tchannel single top-quark production and decay at next-to-next-toleading order in QCD, arXiv:1708.09405 [hep-ph]

27. D. Bardin, S. Bondarenko, L. Kalinovskaya, V. Kolesnikov, W. von Schlippe, Electroweak radiative corrections to single-top production. Eur. Phys. J. C71, 1533 (2011). arXiv:1008.1859 [hep-ph]

28. T. Gleisberg, S. Höche, F. Krauss, M. Schönherr, S. Schumann, F. Siegert, J. Winter, Event generation with SHERPA 1.1. JHEP 02, 007 (2009). arXiv:0811.4622 [hep-ph]

29. S. Frixione, B.R. Webber, Matching NLO QCD computations and parton shower simulations. JHEP 06, 029 (2002). arXiv:hep-ph/0204244

30. S. Höche, F. Krauss, M. Schönherr, F. Siegert, A critical appraisal of NLO+PS matching methods. JHEP 09, 049 (2012). arXiv:1111.1220 [hep-ph]

31. S. Höche, F. Krauss, M. Schönherr, F. Siegert, W + n-jet predictions with MC@NLO in Sherpa. Phys. Rev. Lett. 110, 052001 (2013). arXiv:1201.5882 [hep-ph]

32. S. Höche, M. Schönherr, Uncertainties in next-to-leading order plus parton shower matched simulations of inclusive jet and dijet production. Phys. Rev. D86, 094042 (2012). arXiv:1208.2815 [hep$\mathrm{ph}]$

33. A. Denner, S. Dittmaier, S. Kallweit, S. Pozzorini, NLO QCD corrections to WWbb production at hadron colliders. Phys. Rev. Lett. 106, 052001 (2011). arXiv:1012.3975 [hep-ph]

34. A. Denner, S. Dittmaier, S. Kallweit, S. Pozzorini, NLO QCD corrections to off-shell top-antitop production with leptonic decays at hadron colliders. JHEP 10, 110 (2012). arXiv:1207.5018 [hep-ph]

35. R. Frederix, Top quark induced backgrounds to Higgs production in the $W W^{(*)} \rightarrow l l v v$ decay channel at next-to-leading-order in QCD. Phys. Rev. Lett. 112(8), 082002 (2014). arXiv:1311.4893 [hep-ph]

36. F. Cascioli, S. Kallweit, P. Maierhöfer, S. Pozzorini, A unified NLO description of top-pair and associated Wt production. Eur. Phys. J. C74(3), 2783 (2014). arXiv:1312.0546 [hep-ph]

37. T. Ježo, J .M. Lindert, P. Nason, C. Oleari, S. Pozzorini, An NLO + PS generator for $t \bar{t}$ and $W t$ production and decay including non- 
resonant and interference effects. Eur. Phys. J. C76(12), 691 (2016). arXiv:1607.04538 [hep-ph]

38. S. Catani, M.H. Seymour, A general algorithm for calculating jet cross sections in NLO QCD. Nucl. Phys. B485, 291-419 (1997). arXiv:hep-ph/9605323

39. S. Catani, M.H. Seymour, The dipole formalism for the calculation of QCD jet cross sections at next-to-leading order. Phys. Lett. B378, 287-301 (1996). arXiv:hep-ph/9602277

40. T. Gleisberg, F. Krauss, Automating dipole subtraction for QCD NLO calculations. Eur. Phys. J. C53, 501-523 (2008). arXiv:0709.2881 [hep-ph]

41. F. Krauss, R. Kuhn, G. Soff, AMEGIC++ 1.0: a matrix element generator In C++. JHEP 02, 044 (2002). arXiv:hep-ph/0109036

42. T. Gleisberg, S. Höche, Comix, a new matrix element generator. JHEP 12, 039 (2008). arXiv:0808.3674 [hep-ph]

43. F. Cascioli, P. Maierhöfer, S. Pozzorini, Scattering amplitudes with open loops. Phys. Rev. Lett. 108, 111601 (2012). arXiv:1111.5206 [hep-ph]

44. A. Denner, S. Dittmaier, L. Hofer, COLLIER - a fortran-library for one-loop integrals. PoS LL2014, 071 (2014). arXiv:1407.0087 [hep-ph]

45. G. Ossola, C.G. Papadopoulos, R. Pittau, CutTools: a program implementing the OPP reduction method to compute one-loop amplitudes. JHEP 0803, 042 (2008). arXiv:0711.3596 [hep-ph]

46. A. van Hameren, OneLOop: for the evaluation of one-loop scalar functions. Comput. Phys. Commun. 182, 2427-2438 (2011). arXiv: 1007.4716 [hep-ph]

47. S. Schumann, F. Krauss, A parton shower algorithm based on Catani-Seymour dipole factorisation. JHEP 03, 038 (2008). arXiv:0709.1027 [hep-ph]

48. S. Höche, S. Kuttimalai, S. Schumann, F. Siegert, Beyond standard model calculations with Sherpa. Eur. Phys. J. C75(3), 135 (2015). arXiv: 1412.6478 [hep-ph]

49. D.R. Yennie, S.C. Frautschi, H. Suura, The infrared divergence phenomena and high-energy processes. Ann. Phys. 13, 379-452 (1961)

50. M. Schönherr, F. Krauss, Soft photon radiation in particle decays in SHERPA. JHEP 12, 018 (2008). arXiv:0810.5071 [hep-ph]

51. T. Sjöstrand, M. van Zijl, A multiple-interaction model for the event structure in hadron collisions. Phys. Rev. D36, 2019 (1987)

52. A. De Roeck, H. Jung (eds.), HERA and the LHC: a workshop on the implications of HERA for LHC physics: proceedings part A, CERN, Geneva (2005)

53. J.-C. Winter, F. Krauss, G. Soff, A modified cluster-hadronisation model. Eur. Phys. J. C36, 381-395 (2004). arXiv:hep-ph/0311085
54. S. Höche, F. Krauss, M. Schönherr, F. Siegert, QCD matrix elements + parton showers: the NLO case. JHEP 04, 027 (2013). arXiv:1207.5030 [hep-ph]

55. T. Gehrmann, S. Höche, F. Krauss, M. Schönherr, F. Siegert, NLO QCD matrix elements + parton showers in $e^{+} e^{-} \rightarrow$ hadrons. JHEP 01, 144 (2013). arXiv:1207.5031 [hep-ph]

56. S. Höche, J. Huang, G. Luisoni, M. Schönherr, J. Winter, Zero and one jet combined NLO analysis of the top quark forward-backward asymmetry. Phys. Rev. D88, 014040 (2013). arXiv:1306.2703 [hep-ph]

57. S. Höche, F. Krauss, P. Maierhöfer, S. Pozzorini, M. Schönherr, F. Siegert, Next-to-leading order QCD predictions for top-quark pair production with up to two jets merged with a parton shower. Phys. Lett. B748, 74-78 (2015). arXiv:1402.6293 [hep-ph]

58. R.D. Ball et al., The NNPDF collaboration, Parton distributions for the LHC Run II, JHEP 04, 040 (2015). arXiv:1410.8849 [hep-ph]

59. A. Buckley, J. Ferrando, S. Lloyd, K. Nordström, B. Page, M. Rüfenacht, M. Schönherr, G. Watt, LHAPDF6: parton density access in the LHC precision era. Eur. Phys. J. C75, 132 (2015). arXiv: 1412.7420 [hep-ph]

60. J. R. Andersen et al., Les Houches 2013: physics at TeV colliders: standard model working group report, arXiv:1405.1067 [hep-ph]

61. E. Bothmann, M. Schönherr, S. Schumann, Reweighting QCD matrix-element and parton-shower calculations. Eur. Phys. J. C76(11), 590 (2016). arXiv:1606.08753 [hep-ph]

62. A. Buckley, J. Butterworth, L. Lönnblad, D. Grellscheid, H. Hoeth et al., Rivet user manual. Comput. Phys. Commun. 184, 2803-2819 (2013). arXiv:1003.0694 [hep-ph]

63. M. Cacciari, G.P. Salam, G. Soyez, The Anti-k(t) jet clustering algorithm. JHEP 04, 063 (2008). arXiv:0802.1189 [hep-ph]

64. M. Cacciari, G.P. Salam, G. Soyez, The catchment area of jets. JHEP 04, 005 (2008). arXiv:0802.1188 [hep-ph]

65. V. Bertone, S. Carrazza, J. Rojo, APFEL: a PDF evolution library with QED corrections. Comput. Phys. Commun. 185, 1647-1668 (2014). arXiv:1310.1394 [hep-ph]

66. S. Alekhin, J. Blümlein, S. Moch, R. Plačakytè, Isospin asymmetry of quark distributions and implications for single top-quark production at the LHC. Phys. Rev. D94(11), 114038 (2016). arXiv:1508.07923 [hep-ph]

67. S. Alekhin, J. Blumlein, S. Moch, Parton distribution functions and benchmark cross sections at NNLO. Phys. Rev. D86, 054009 (2012). arXiv:1202.2281 [hep-ph]

68. L.A. Harland-Lang, A.D. Martin, P. Motylinski, R.S. Thorne, Parton distributions in the LHC era: MMHT 2014 PDFs. Eur. Phys. J. C75(5), 204 (2015). arXiv:1412.3989 [hep-ph] 